\title{
A Nonlinear Technique for Automatic Twin-Image and Zero-Order Term Suppression in Digital Holographic Microscopy
}

\author{
Nicolas Pavillon ${ }^{1}$, Chandra Sekhar Seelamantula ${ }^{2}$, Michael Unser ${ }^{2}$, and \\ Christian Depeursinge ${ }^{1}$ \\ ${ }^{1}$ Advanced Photonics Laboratory, ${ }^{2}$ Biomedical Imaging Group, Ecole \\ Polytechnique Fédérale de Lausanne (EPFL), 1015 Lausanne, Switzerland
}

\section{Introduction}

Digital holography enables the encoding of complex wavefronts with an intensity measurement, making it possible to recover the quantitative phase for phase-contrast imaging [1] or profilometry [2]. Encoding the wavefront in this fashion leads, however, to the generation of artifacts such as the socalled zero-order, which is the sum of the intensities of the object and reference waves, and the so-called twin-image, which corresponds to the complex conjugate of the desired imaging order.

Since the first developments, efforts have been made to suppress the artifacts either by separation in the spatial Fourier domain in the so-called off-axis configuration [3], or by temporal separation through phaseshifting algorithms [4]. The spatial separation method has the advantage of making it possible to reconstruct the hologram from a single measurement, but at the cost of loss of bandwidth for the imaging terms.

We propose here a technique based on non-linear filtering to suppress the zero-order artifact for off-axis digital holographic microscopy (DHM). The advantages of the proposed method are: (1) it can potentially increase the spatial bandwidth for the imaging terms; (2) it is non-iterative; and (3) it keeps the one-shot feature of DHM.

\section{Principle}

The technique is based on the use of a non-linear operator during hologram filtering. The fundamental equation of interference on a detector is:

$$
i=(r+o)(r+o)^{*}=|o|^{2}+|r|^{2}+r^{*} o+r o^{*},
$$


where all functions are defined in the $(x, y)$ plane, $r$ is the reference wave, $o$ is the object wave, and $(*)$ denotes the complex conjugate.

By rearranging the terms in Eq. 1, and using the logarithm operator, we get that:

$$
\ln \left[\frac{i}{|r|^{2}}\right]=\ln \left[1+\frac{o}{r}\right]+\ln \left[\left(1+\frac{o}{r}\right)^{*}\right] .
$$

It can be shown that under the condition that the reference intensity is stronger than that of the object, the spectral supports of the two terms on the right-hand side of Eq. 2 are non-overlapping. This implies that the wavefront reconstruction method based on Fourier filtering [5] can be employed, by applying it to the so-called cepstrum domain [6]. The reconstruction equation for the object wavefront becomes:

$$
\begin{aligned}
& o^{\prime}(x, y)=C \cdot D(x, y) \cdot\left[\exp \left(i_{F}^{\prime}\right)-1\right], \\
& i_{F}^{\prime}=F^{-1}\left\{F\left\{\ln \left[\frac{i(x, y)}{|r(x, y)|^{2}}\right]\right\} \cdot W\left(\omega_{x}, \omega_{y}\right)\right\},
\end{aligned}
$$

where $F$ is the Fourier transform operator, $C$ is a real constant, and $W\left(\omega_{x}, \omega_{y}\right)$ is a window function in the spectral domain. The variable $D(x, y)$ is a phase mask for hologram demodulation.

One can observe from Eq. 3 that the knowledge of the reference intensity is required. It is either possible to use a measurement of the reference by blocking the object wave, or by employing a model such as a plane wave as an estimator of the reference wave. For practical reconstruction, a discrete version of Eq. 3 is implemented.

\section{Experimental results}

In order to demonstrate the performance of the method, we made measurements on a transmission DHM setup, employing a Mach-Zehnder interferometer. The sample, a solution containing yew pollens, is illuminated with a laser diode $(\lambda=652.5 \mathrm{~nm})$, and the image is recorded in the Fresnel zone with an 8-bit charge-coupled device (CCD) camera ( $\Delta x=6.45 \mu \mathrm{m}$ ) with $10 \mathrm{x}$ magnification. The hologram is reconstructed with the standard spatial filtering method applied to a quadrant of the image, as well as with the non-linear technique. The complex wavefront is 
then digitally propagated into focus $(d=4.4 \mathrm{~cm})$ with a numerical implementation of the Fresnel integral.
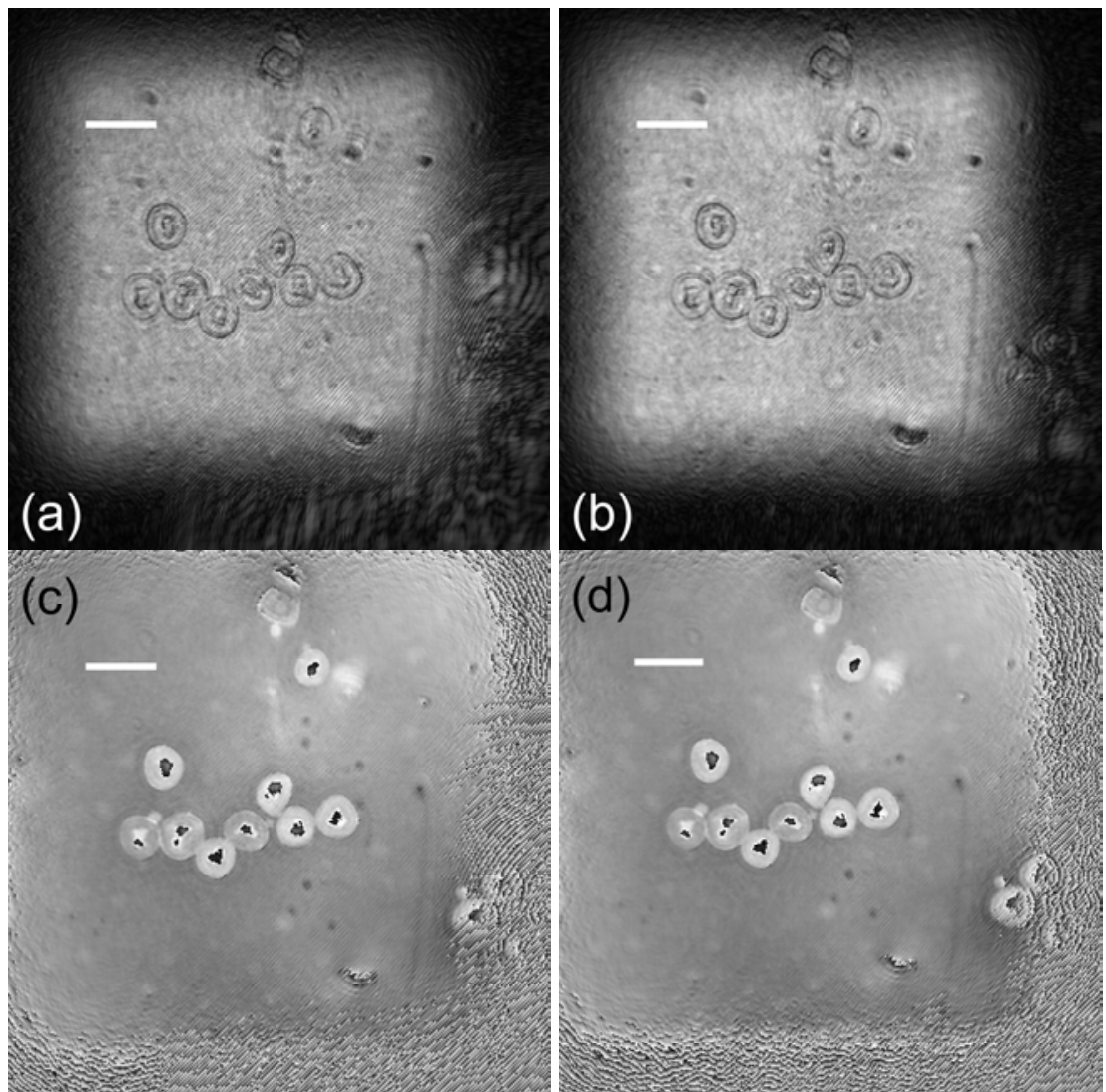

Fig. 1. Image of a yew pollens hologram reconstructed with (a, c) standard spatial filtering and (b, d) non-linear method, where artefacts at the bottom right are suppressed both in amplitude (a, b) and in phase (c, d) images. The scale bars are $20 \mu \mathrm{m}$ long.

The intensity ratio is controlled by using a neutral density filter in the path of the object arm of the interferometer. The mean of the reference intensity divided by the object intensity in this case is 12.1. The reconstructions are shown in Fig. 1, for the standard method (Fig. 1.(a, c)) and the non-linear technique (Fig. 1.(b, d)), respectively. The suppression of the residues of the zero-order can be readily identified in both amplitude (Fig. 1.(a, b)) and phase (Fig. 1.(c, d)) at the bottom-right of the images. Some remnants of the zero-order are still present in the reconstruction, because of quantization and measurement noise. 


\section{Conclusion}

We presented the principle of a new non-linear filtering method for artifact-free reconstruction in digital holography. This method intrinsically suppresses the so-called zero-order, and thus increases the available bandwidth for the imaging orders in off-axis holography. We provided experimental results to validate the technique.

\section{Acknowledgements}

N. Pavillon and C. Depeursinge were supported by the Swiss National Science Foundation (SNSF) grant \#205320-120118. C.S. Seelamantula and $\mathrm{M}$. Unser were supported by the Center for Biomedical Imaging (CIBM) of the Geneva-Lausanne universities and the EPFL, Hasler, Leenards, and Louis-Jeantet foundations. The authors would like to thank their colleagues in the MVD Group and at Lyncee Tec SA (www.lynceetec.com) for their cooperation and fruitful discussions.

\section{References}

1. Marquet, P, Rappaz, B, Magistretti, P J, Cuche, E, Emery, Y, Colomb, T, Depeursinge, C (2005) Digital holographic microscopy: a noninvasive contrast imaging technique allowing quantitative visualization of living cells with subwavelength axial accuracy. Optics Letters 30:468-470

2. Schnars, U, Jüptner, W P O (2002) Digital recording and numerical reconstruction of holograms. Measurement Science and Technology 13:R85-R101

3. Leith, E N, Upatnieks, J (1962) Reconstructed Wavefronts and Communication Theory. Journal of the Optical Society of America 52:1123-1130

4. Yamaguchi, I, Zhang, T (1997) Phase-shifting digital holography. Optics Letters 22:1268-1270

5. Cuche, E, Marquet, P, Depeursinge, C (1999) Simultaneous amplitudecontrast and quantitative phase-contrast microscopy by numerical reconstruction of Fresnel off-axis holograms. Applied Optics 38:69947001

6. Oppenheim, A V, Schafer, R W (2004) From frequency to quefrency: a history of the cepstrum. Signal Processing Magazine, IEEE 21:95-106 